\title{
Transillumination of iris and subnormal visual acuity_ocular albinism?
}

Eye Clinic, Mölndals Hospital, Mölndal, Sweden

L Sjödell

Pediatric Eye Clinic, East Hospital, Sweden A Sjöström

Department of Ophthalmology, Göteborg University, Göteborg, Sweden M Abrahamsson

Correspondence to: Dr Lena SjödeIl, Eye Clinic, Mölndals Hospital, S-431 80 Mölndal, Sweden.

\begin{abstract}
Background-A common clinical sign in children with subnormal visual acuity or slow visual development was iris transillumination. This was used as the inclusion criterion in a study of children shown to have a subnormal visual acuity in a general health examination at age 4 years. Methods-Refraction values, stereopsis, fundus photography, macular and nerve head appearance, and visual evoked response (VER) recordings were studied in 18 children. The clinical results were compared with 64 controls referred to the eye clinic because of subnormal vision from the general health examination or from school health care.
\end{abstract}

Results-Eight children had VERs showing asymmetry typical for albinism. Another four had only small asymmetries on the VER, indicating a lower degree of decussation abnormality. No simple correlation of visual acuity, degree of iris transillumination, stereopsis, or macular pathology and VER asymmetries were found. However, marked iris transillumination in all four quadrants, absence of a foveal reflex, and low visual acuity were weakly correlated.

Conclusions-In a rather homogeneous group of children with iris transillumination and subnormal visual acuity eight of 18 had typical albino VERs. The findings of small atypical VER asymmetries in four children and no asymmetry in six children suggest that albinism may be considered as a description of a heterogeneous group of conditions including maximal decussation rate $(100 \%)$ in the chiasma to a condition with almost normal ( $\geqslant 50 \%)$ decussation rate.

(Br f Ophthalmol 1996;80:617-623)

Albinism is usually characterised by nystagmus, foveal hypoplasia, photophobia, iris translucency, neuronal abnormalities, and decreased visual acuity, ${ }^{1-6}$ but manifestations may vary considerably and albinism may be diagnosed only as a result of a subnormal visual acuity, or by chance. ${ }^{78}$ Albinism is a genetically determined disorder of the melanin pigmentary system ${ }^{5}{ }^{10}$ which gives a hypopigmentation of skin, hair, and eyes (oculocutaneous albinism, OCA) or a hypopigmentation of the eyes only (ocular albinism, OA). The melanocytes of the pigment epithelial layers of the retina, ciliary body, and iris are derived from the outer layer of the optic vesicle (neuroectoderm). This melanogenesis occurs early, already by the fifth week of fetal life, and it is thought to be completed at or soon after birth. ${ }^{11}$ By the ninth gestational week there is a relative hyperpigmentation of the macula. The pigmentation of skin, hair, and parts of the anterior uveal tract originates from melanocytes derived from the neural crest cells and appears at about the 20th gestational week. ${ }^{5}$ The optic pathways from eye to chiasma form early, before there is any pigment in the mammalian embryo except for the retinal pigment and a transient pigmentation of the upper wall of the distal primitive eye stalk. $^{3}{ }^{11} 12$ In normal embryos, the development of the optic nerve and chiasmal decussation seems to depend on the development of melanin and pigmented cells. ${ }^{3}$ In albinism, the lack of pigment cells and melanin of the retina is thought to play a major role in the misrouting of the optic nerve axons at the chiasma. The foveal pigmentation is, in the same way, thought to be essential to the normal development of the foveal photoreceptors and visual resolution..$^{3-5}{ }^{13}$ Albinos have an optic chiasm, where temporal retinal fibres that normally should remain ipsilateral decussate to the contralateral side. The relatively larger number of fibres decussating compared with the number remaining on the ipsilateral side will give asymmetric visual evoked responses (VER) from the ipsilateral compared with the contralateral visual cortex after monocular stimulation. $^{3}{ }^{14}$ It has been claimed that this increase in the number of decussating fibres can be shown with VER with a sensitivity of $100 \%$ and are pathognomonic for albinism. ${ }^{14}$ However, asymmetric VER has been detected in patients with dissociated vertical deviation, ${ }^{15}$ although this finding is controversial. ${ }^{16}$ Asymmetric VERs have also been recorded in cases of chiasmal malformations but in the opposite direction compared with albinism. ${ }^{17}$

Albinoidism refers to another group of patients with congenital hypomelanosis but without nystagmus, neuronal, or other abnormality and with normal or only rarely subnormal visual acuity. ${ }^{5}$

Optic malformations (hypoplasia, atrophy, and coloboma) and retinal dystrophies may result in subnormal vision, and in some cases these anomalies will remain undetected for a long time. In other cases low vision can already be detected in the infant (DVM, delayed visual maturation), although the causes may be obscure. Thus, such conditions (DVM, albinism, albinoidism, retinal dystrophies, and optic malformation) may be very difficult to 
diagnose, and can be referred to as subnormal visual acuity syndromes (SVAS) in children. Since they have a somewhat different prognosis, and because SVAS may also be the first sign of a neuroimpairing condition, it is of great importance to be able to diagnose and give a prognosis of the condition. This paper describes such a group of children with SVAS who as a result of subnormal visual acuity were referred to the eye clinic. They had no obvious clinical signs indicating a particular syndrome or condition, except iris transillumination which suggested an ocular albinism.

\section{Subjects and methods}

We studied children who were referred to the eye clinic in Mölndal because of early strabismus or subnormal visual acuity at a visual screening included in a general health control of all children aged 4 years in Sweden. ${ }^{18}$ Informed consent from both children and parents was obtained for patients and controls. Four children were sent to the eye clinic because of subnormal acuity in the test at school. The inclusion criterion has been iris transillumination, which was recognised at the clinical examination performed when visual acuity did not improve as expected. Apparent nystagmus has been an exclusion criterion since these children often have an early diagnosis of albinism. The aim of this study was to examine a group of children where the manifestations of possible albinism were more subtle and to try to find out if an ocular albinism could explain the subnormal visual acuity. The children were followed clinically for 2 to 11 years and they have been treated with glasses and occlusion according to the general regime in our clinic. No severe ocular malformations or retinal dystrophies were found.

The children were called in for a standard examination. The group included 18 children, five boys and 13 girls, and at the last examination they were aged 6-21 years (mean age 12.4 years, median age 12 years). The examination included iris translucency grading, refraction values, best corrected visual acuity, assessment of strabismus and nystagmus, best stereoacuity tested with TNO, Titmus fly or Bagolini, and VER recordings.

\section{VISUAL ACUITY}

A letter chart at 5 metres distance was used to test the visual acuity. When the child was too young to read letters an tumbling $E$ chart at the same distance was used. A maximum of two erroneous identified letters on a single row was allowed.

\section{IRIS TRANSLUCENCY}

Iris transillumination was accomplished by routine slit-lamp examination ${ }^{19}$ with only one desklamp lit in the otherwise darkened room. The transillumination was graded in the following manner: minimal defects (I), moderate defects but lens equator not seen (II), moderate defects and lens equator visible (III), or obvious defects and lens equator clearly seen (IV). Furthermore, there was an assessment as to whether the transillumination engaged the periphery of the iris, the centre, or both and how many quadrants of the iris showed transillumination. Two observers made this examination independently in 15 of the 18 patients to test the reproducibility of the method. The agreement between the observers was good and only minor disagreements were seen in a few cases.

\section{REFRACTION, MACULAR EXAMINATION, FUNDUS} PHOTOGRAPHY

Autorefraction and ophthalmoscopy were performed after inducing pupillary dilatation and cycloplegia with cyclopentolate eyedrops.

Fundus photography was also included, and special interest was focused on the macula during examination of the photographs. This examination was performed independently by three ophthalmologists. Two observers also made an independent assessment of the pigmentation of the fundus. The visibility of the choroidal vasculature in the four quadrants of the fundus and in the posterior pole were scored. A maximal score was 17 points when the choroidal vessels were easily seen all over the fundus and 0 points were scored if the whole fundus was well pigmented.

\section{VISUAL EVOKED RESPONSE, VISUAL EVOKED}

\section{POTENTIALS}

For the VER recordings a visual stimulus of a short latency light flash of high intensity (supramaximal) was used (Grass PS 22, intensity setting 16 , duration $<100 \mu \mathrm{s}$ ), bi- and monocularly in each session. Particular attention was paid to accomplish a complete cover of the fellow eye in monocular stimulation. Recordings from five positions in a horizontal row at the occipital level ${ }^{14}$ were performed (Ola, O1, Oz, O2, O2a). The VER activity was averaged $(n=20-30)$ by a Medelec average oscilloscope, with bandpass filter of $2-200 \mathrm{~Hz}$. The VERs were manually analysed by two experienced VER scientists, particularly with respect to side asymmetries to monocular stimulation. ${ }^{814}$ In each session two series of recordings were performed to control consistency of the response. The recordings from two sessions were analysed for 10 subjects, and from one session for seven subjects. One patient did not turn up at any session.

\section{CONTROL SUBJECTS}

A group of 64 patients ( 32 boys and 32 girls) served as controls concerning the ophthalmic examination (refraction, visual acuity, iris transillumination, retinal inspection). These were consecutive patients sent to us because of subnormal vision at the 4 year health control mentioned above or from the school health care. Some of these children have only been to the eye clinic once for examination while others have been followed and treated with glasses and/or occlusion. At the last examination they were aged 4-10 years (mean age 6.2, median age 6 years).

Twelve children, 4 to 12 years old, with normal visual acuity and no iris transillumination volunteered for the electrophysiological examination. 
Table 1 Characteristics of study group

\begin{tabular}{|c|c|c|c|c|c|c|c|c|c|c|c|c|}
\hline \multirow{2}{*}{$\begin{array}{l}\text { Sex/year } \\
\text { of birth }\end{array}$} & \multicolumn{2}{|c|}{ Visual acuity } & \multirow[b]{2}{*}{ Iris } & \multirow[b]{2}{*}{ Macula } & \multicolumn{2}{|c|}{ Visual evoked potential } & \multirow[b]{2}{*}{ Stereo } & \multirow[b]{2}{*}{ Strabismus } & \multirow[b]{2}{*}{ Iris colour } & \multirow[b]{2}{*}{ Tan } & \multirow{2}{*}{$\begin{array}{l}\text { Fundus } \\
\text { scale } 0-17\end{array}$} & \multirow[b]{2}{*}{ Treatment } \\
\hline & 1991 & 1993 & & & 1992 & 1993 & & & & & & \\
\hline Female/84* & $0.6 / 0.4$ & $0.7 / 0.5$ & IV $/ 4 c$ & - & Sym & Sym & $60^{\prime \prime}$ & $\mu$ eso & Mixed & Slightly tanned & $14 / 13.5$ & $\mathrm{~S}+\mathrm{O}$ \\
\hline Female/82 & $0.5 / 0.5$ & $0.5 / 0.4$ & IV $/ 4 c$ & - & Not sym & Not sym & $800^{\prime \prime}$ & Eso & Mixed & Red & $6.5 / 6.5$ & \\
\hline Female/79† & $0.3 / 0.5$ & $0.4 / 0.5$ & $\mathrm{IV} / 4 \mathrm{c}$ & - & Sym & & +Bagolini & Eso & Blue & Slightly tanned & $7.5 / 8.0$ & $\mathrm{~S}+\mathrm{O}$ \\
\hline Female/77 $\dagger$ & $0.7 / 0.5$ & $0.7 / 0.7$ & $\mathrm{IV} / 4 \mathrm{c}$ & - & Not sym & & Titmus & 0 & Blue & Slightly tanned & $9.5 / 11.5$ & None \\
\hline Female/81 & $0.6 / 0.9$ & $0.6 / 0.9$ & IV/4 & + & Not sym & Not sym & Titmus & 0 & Mixed & Slightly tanned & $4.5 / 3$ & $\mathbf{S}$ \\
\hline Male/81 & $0.6 / 0.3$ & $0.6 / 0.5$ & IV $/ 4$ & - & & & +Bagolini & Exo & Mixed & Slightly tanned & $11 / 12.5$ & $S+O$ \\
\hline Female/87^ & $0.5 / 0.5$ & $0.6 / 0.7$ & IV $/ 4$ & - & Not sym & Not sym & $60^{\prime \prime}$ & 0 & Blue & Slightly tanned & $16.5 / 16.0$ & None \\
\hline Female/73 & & $0.7 / 0.7$ & IV $/ 4$ & - & Not sym & & $240^{\prime \prime}$ & 0 & Blue & Tanned & $10.0 / 12.0$ & None \\
\hline Male/76‡ & $0.9 / 1.0$ & & IV/4 & + & ns & & $60^{\prime \prime}$ & Intermitt exo & Mixed & Slightly tanned & $3.5 / 3.0$ & S \\
\hline Male/71 & $0.7 / 0.9$ & & IV $/ 2$ & + & & & & 0 & Blue & Slightly tanned & $8.5 / 8.5$ & S \\
\hline Female/83‡ & $0.7 / 0.9$ & $1.0 / 1.0$ & IV/2 & + & Not sym & Not sym & $30^{\prime \prime}$ & 0 & Mixed & Slightly tanned & $4.5 / 4$ & $\mathrm{~S}+\mathrm{O}$ \\
\hline Female/86 & $0.5 / 0.4$ & $0.7 / 0.7$ & IV/2 & + & Not sym & Not sym & $120^{\prime \prime}$ & 0 & Mixed & Tanned & $1 / 1$ & $S+F$ \\
\hline Male/79 & $0.7 / 0.9$ & $0.7 / 1.0$ & III/3 & + & ns & ns & $240 "$ & 0 & Blue & Red & $10 / 8.5$ & S \\
\hline Female/81 & $0.7 / 0.7$ & $0.9 / 0.9$ & III $/ 3$ & + & ns & & $60^{\prime \prime}$ & 0 & Blue & Tanned & $4.5 / 5.5$ & $\mathbf{S}$ \\
\hline Female/81 & $0.8 / 0.5$ & $1.0 / 0.5$ & III/1 & + & & Sym & $40^{\prime \prime}$ & 0 & Blue & Slightly tanned & $5.5 / 5.5$ & S \\
\hline Male/82 & $0.7 / 0.7$ & $0.7 / 0.7$ & II/2 & + & Sym & Sym & Titmus & $\mu$ eso & Blue & Tanned & $1.5 / 2$ & $s+O$ \\
\hline Female/79 & $1.0 / 1.0$ & $1.0 / 1.0$ & II $/ 2$ & + & & Sym & $240^{\prime \prime}$ & 0 & Blue & Red & 9/7.5 & None \\
\hline Female/83 & $0.8 / 0.5$ & $0.8 / 0.6$ & II $/ 1$ & + & & Not sym & $60^{\prime \prime}$ & 0 & Blue & Red & $10.5 / 10.5$ & $S+O$ \\
\hline
\end{tabular}

$\mathrm{S}=$ spectacles, $\mathrm{O}=$ occlusion, $\mathrm{F}=$ filter; II-IV=grade of transillumination; $1-4=$ number of quadrants engaged by transillumination; $\mathrm{c}=\mathrm{centre}$ of iris transilluminated; $\star=$ sisters, $\dagger=$ sisters, $\ddagger=$ cousins; Macula $+=O K,-=$ no foveal reflex; Fundus scale 0 all of fundus pigmented, 17 no pigment in fundus; Sym=symmetric VEP; Not sym=asymmetric VEP; ns=small asymmetry, see text.

\section{STATISTICS}

Correlation coefficients were calculated for the different relations between degree of iris transillumination, stereopsis, visual acuity, VER asymmetries, foveal reflex, and visual acuity.

\section{Results}

The results of the SVAS group are summarised in Table 1.

VISUAL ACUITY (FIGS 1A AND 1B)

The mean visual acuity for the study group was 0.73 (range 0.4-1.0) compared with 0.85 for the control group (range $0.2-1.0$ ). In the control group there are two eyes with acuity below 0.6 -in the eye with acuity 0.2 the occlusion therapy was not accomplished, and the eye with acuity 0.5 was highly myopic $(-9 \mathrm{D})$ and amblyopic in spite of spectacles and occlusion therapy. Best corrected visual acuity in the better eye was 0.8 or more in $50 \%$ of patients in the study group and in $87.5 \%$ in the control group.

REFRACTION

The spherical errors in the study group ranged from -5.25 D to +7.5 D; seven myopic eyes, 29 hyperopic eyes, but no emmetropic eye. In the control group the range was from $-9 \mathrm{D}$ to +6.75 D; 12 myopic, four emmetropic, and 112 hyperopic eyes. If we disregard the patient with monocular myopia of $9 \mathrm{D}$, the most myopic eye in the control group was $-3.25 \mathrm{D}$.

There was a higher incidence of astigmatism in the study group. Fifty per cent of the patients $(42 \%$ of the eyes) had a cylinder of 1-3 D compared with $34 \%$ of the patients ( $25 \%$ of the eyes) in the control group.

Anisometropia, defined as $1 \mathrm{D}$ difference or more in the most refracting axis, was more common in the study group (50\%) than in the control group (14\%). If the anisometropia is defined as $1 \mathrm{D}$ difference or more, in spherical equivalents the corresponding figures are $44.5 \%$ and $10.9 \%$ respectively. The frequency of anisometropia in the control group is higher than expected in normal individuals. Among children the prevalence is reported to be $1.4-4.7 \%^{16} 1722$ and in an adult population $9 \%,{ }^{21}$ but there are differences in the definition of anisometropia between the authors. The higher anisometropia prevalence in our control group is explained by the referral system (see Subjects and methods).
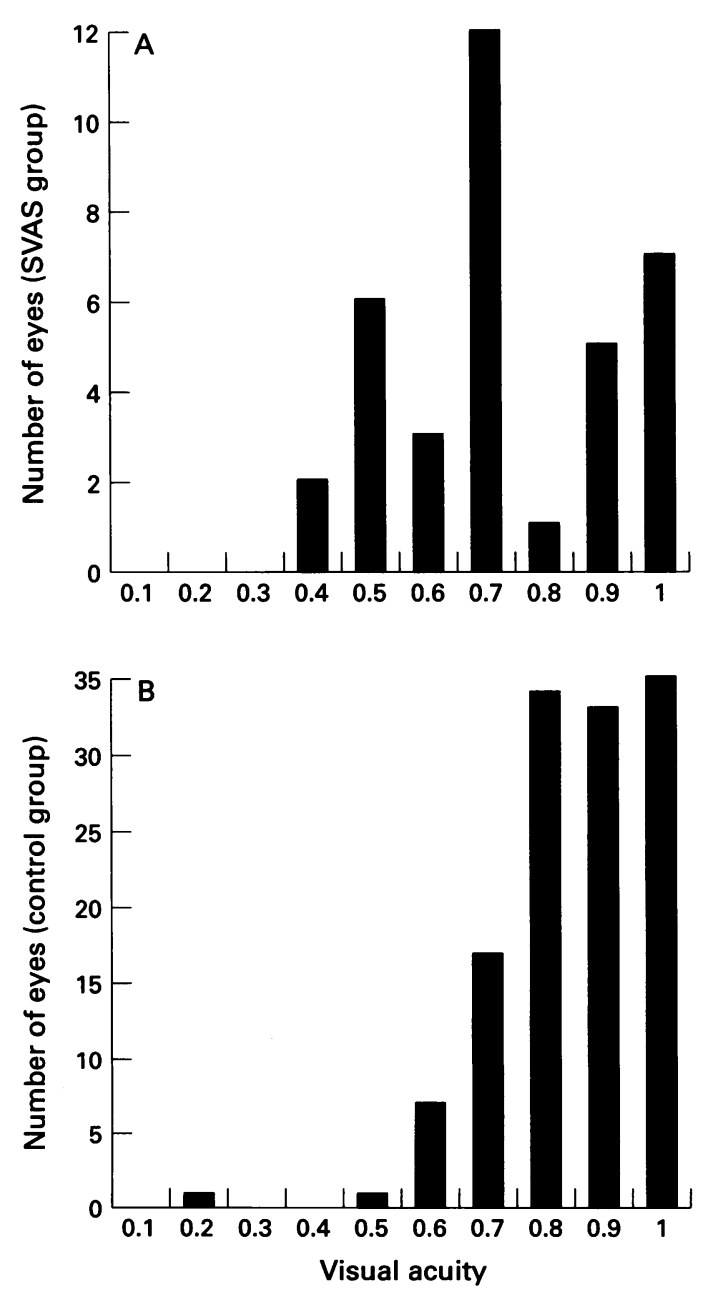

Figure 1 (A) Visual acuity of the group with subnormal visual acuity syndrome (SVAS) (18 children, 36 eyes). (B) Visual acuity of the control group (64 children, 128 eyes). 
PIGMENTATION OF SKIN, HAIR, AND IRIDES Normal tan was self reported in four patients while a slight tan was self reported in 10 patients. In four cases sunlight exposure results in a red skin. In the latter case only one patient had an iris transillumination grade IV in all quadrants. The colour of the irides was blue in 11 cases and mixed in seven patients. In no case was there a brown iris. In the group of nine patients with grade IV transillumination in all quadrants there were five patients with mixed iris colour and four with blue irides. The colour of the hair was light brown in two patients both with grade IV transillumination in two quadrants and a non-symmetric visual evoked potential (VEP) on two occasions. The rest of the patients were fair but not extremely blond.

IRIS TRANSILLUMINATION (FIGS 2A AND 2B) All eyes in the study group had iris transillumination since this was the inclusion criterion. The grade of transillumination was variable however (Fig 2A).

Nine children had a marked transillumination with the lens equator clearly seen in all
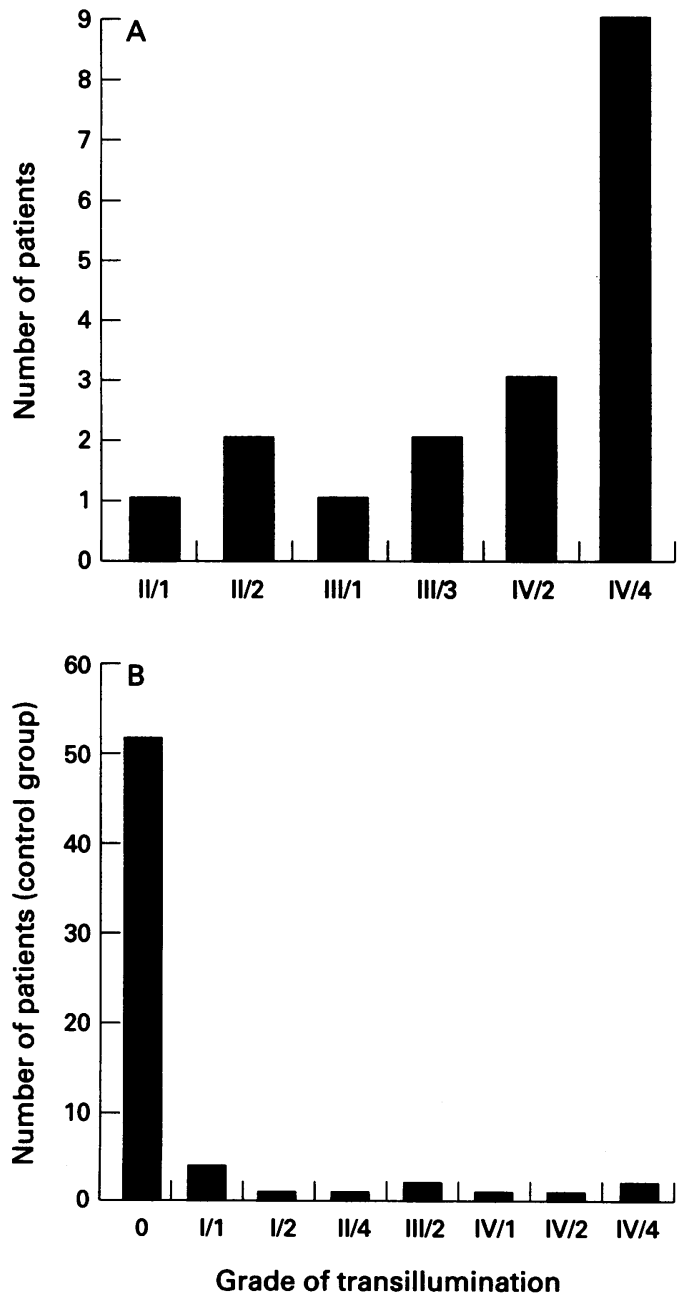

Figure 2 (A) Iris transillumination of the group with subnormal visual acuity syndrome (SVAS). See text for details. II-IV indicate grade of transillumination and 1-4 the number of quadrants engaged by transillumination. The four patients with peripheral and central transillumination are indicated by a stippled area. (B) Iris transillumination of the control group. See $(A)$ and text for details. quadrants (grade IV). Four of them also had clear defects in the pigment in the centre of the iris.

Three patients had grade IV transillumination in two quadrants. Three patients had grade III and three patients grade II in 1-3 quadrants.

Only patients with grade IV showed defects in the centre of the iris. In all patients the iris transillumination was symmetrical in the two eyes.

In the control group (Fig 2B) 12 individuals (18\%) showed some iris transillumination, as even a minute area of transillumination was considered positive in this study. In blue eyed patients eight $(18 \%)$ were positive, in mixed colour eyed patients three ( $30 \%)$ were positive, and in brown eyed patients one $(10 \%)$ was positive. These figures are comparable with the normal transillumination incidence in a population described by Jay et $a l .^{4}$ They found an overall incidence of $9.2 \%$ and $19 \%$ in blue eyed subjects.

Five individuals had grade I transillumination, one individual grade II, two individuals grade III, and four individuals grade IV (Fig 2B). In the individual with grade II transillumination all quadrants were engaged and this was also the case with two individuals with grade IV. In the other individuals 1-2 quadrants had transillumination. All but one individual had symmetrical transillumination in both eyes. This individual had grade I transillumination in one eye and none in the other.

\section{STRABISMUS}

Strabismus was seen in six patients $(33 \%)$ in the study group (Table 1) and in the same number in the control group ( $9 \%)$. In the study group four children had esotropia, one of them is straight with spectacles and has full stereo acuity (60", TNO). Two children had intermittent exotropia.

Among the control children four had esotropia - one is straight with spectacles, one had exotropia, and one hypertropia.

STEREOPSIS (TABLE 1)

Stereoacuity was good: at least 120", in eight children in the study group; moderate, 240 " in three children; and bad, Titmus fly and/or Bagolini positive in six children (three of them have esotropia and one intermittent exotropia). One patient was not tested for stereo acuity.

MACULAR EXAMINATION

In the assessment of the fundi special attention has been focused on the macula. In the study group seven patients had clearly pathological maculas with an indistinct or absent foveal reflex. Eight patients were considered having normal maculas and in one patient all three observers considered the right foveal reflex abnormal while the left fovea was considered normal. In this patient's right eye there is also a pigmentation of unknown aetiology close to the papilla. In two patients the observers differed somewhat in their assessment but in these patients there was no obvious macular pathology. Most of the patients had enough 
pigment in the posterior pole to mask most of the choroidal vasculature but in three patients the choroidal vessels were easily seen. In the control group all patients' maculas were considered normal at clinical examination. Concerning the pigmentation of the fundus there were five patients with a score of 11 or more. They all had a pronounced transillumination of the iris and a pathological macula. The remaining scores did not correlate with iris transillumination or macular pathology. MOTILITY AND NYSTAGMUS

Nystagmus or motility disorders was not a common sign (see Subjects and methods) and only a very subtle nystagmus was noticed in two patients in the study group. One patient had rotatory nystagmus seen in the microscope and the other had an intermittent rotatory nystagmus noticed at some appointments.
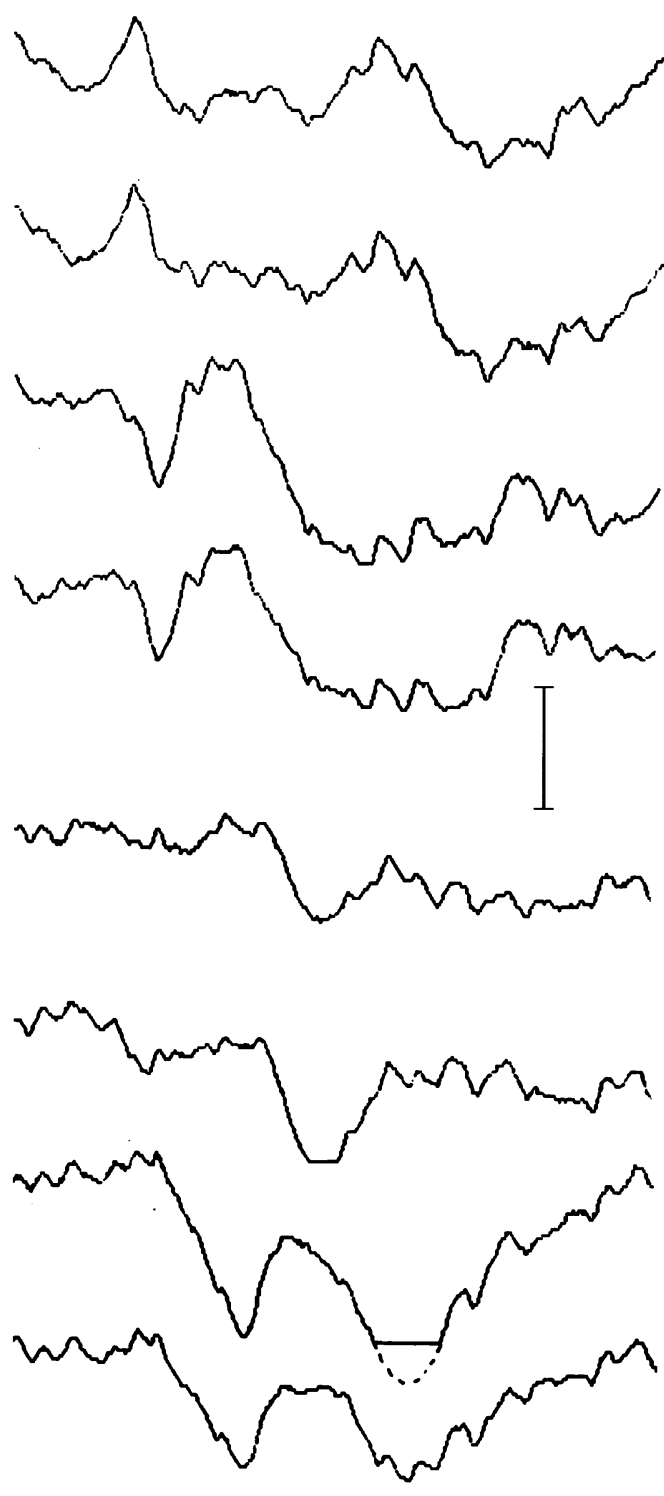

Figure 3 Visual evoked response (VER) from a patient in the group with subnormal visual acuity syndrome. The upper 4VERs are simultaneously recorded from 01

(uppermost), O1a, O2a, and O2-that is, from left to right at the $\mathrm{Oz}$ level. Flash light stimulation of the right eye. The lower 4VERs are responses after stimulation of the left eye.

Note asymmetries and potential reversals after right compared with left eye stimulation. Time base $30 \mathrm{~ms}$ amplification bar $5 \mathrm{nV}$.

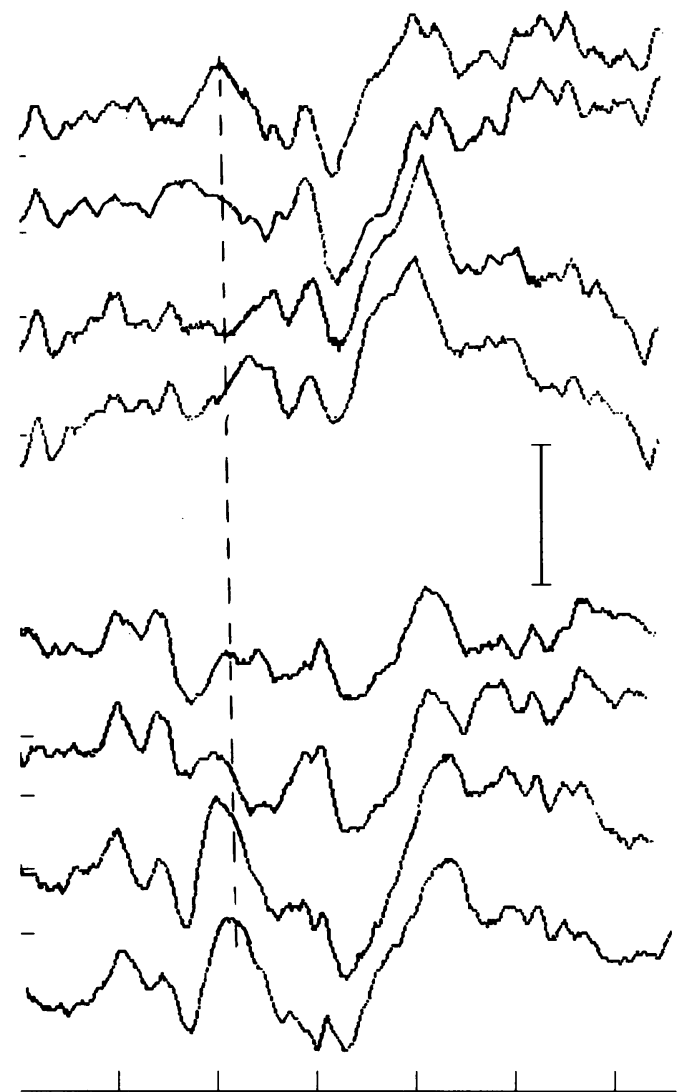

Figure 4 Visual evoked responses (VERs) showing localised asymmetries. Procedures as described in Figure 3 and text. The short latency responses are symmetrical. With onset, as indicated by the broken line, a short period of the VER shows asymmetry, indicating albinoic visual pathways. This kind of response was recorded in some of the patients in the study group, but also, as in this figure from a parent. This VER pattern was recorded from the mother of the patient in Figure 3.

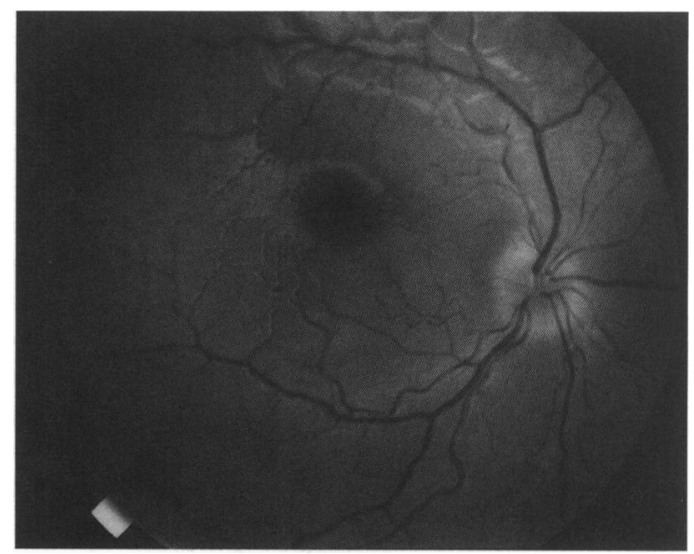

Figure 5 Fundus photograph of a patient with normal pigmentation but asymmetric VER.

VISUAL EVOKED RESPONSE (FIGS 3 AND 4, TABLE 1)

Typical albinoic VER asymmetry was shown in eight patients (Fig 3); five had symmetric VERs and in four patients VER recordings were difficult to classify (Fig 4). In this group the asymmetry was not as distinct as in the typical cases. All of the control VERs were symmetric.

Asymmetry was found among those patients with marked iris transillumination (grade IV) and absent foveal reflex as often as among patients with moderate transillumination and normal macula (Fig 5). Among the patients 
with symmetric VER there were two with marked transillumination and indistinct foveal reflex.

\section{Discussion}

The present study was initiated to investigate unclear subnormal visual acuity and late visual development in preschool children and children in their first school year. In one group we found iris transillumination as a common denominator which could suggest an ocular albinism and thereby explain subnormal visual acuity. We decided to examine this group further. In cases of typical albinism (nystagmus, absent foveal reflexes, very low visual acuity, absence of stereopsis) the diagnostic dilemma did not occur. The aim of the present study was to evaluate the albino diagnosis in more subtle cases.

We found some signs that could indicate ocular albinism. A marked iris transillumination and absence of foveal reflex was found in seven patients and this seems to correlate with a subnormal visual acuity (cf Abadi and Pascal ${ }^{12}$ ). However we have not been able to prove that these patients are albinos in the sense that they do not all have an asymmetric VERs. On the other hand some patients with less iris transillumination, with a normal macula, but subnormal visual acuity did show asymmetric VERs. It seems obvious that a high degree of iris transillumination and ill defined low pigmented maculae/fovea indicate a lack of pigmented cells or low pigment content within pigmented cells. Why such eyes, with clear indications of albinism, do not always have a chiasmal abnormality with an increased number of optic axons decussating is unclear.

In previous studies an electrode montage used as in the present work was claimed to be necessary to detect albinism. ${ }^{13}$ Others claim that only a pair of electrodes, bipolarly connected symmetrically on each side of the occiput, is sufficient for the diagnosis of albinism. ${ }^{28}$ It has also been claimed that in small children the flash evoked VER is a sufficient stimulus to reveal albinism, but that in older children and adults the pattern onset method is preferred. ${ }^{213}$ However, in the present study we recorded albino asymmetries in both teenagers and older children as well as in smaller children. It was also shown recently that, for example, it is possible to record and identify pattern response after a patterned flash stimulus. ${ }^{22}$ Even if the cortical activation differs with different stimuli, a powerful flash stimulus probably activates the main primary visual afferent fibres and cortical neurons. ${ }^{23}{ }^{24}$ Hence, there should be no main afferent fibres silent after a supramaximal flash, as was used in the present study. It should therefore be possible to detect any occipital side differences to monocular stimulation, if there are retinocortical side differences as a result of an albinoic decussation. The detection level and resolution of the method depends not only on the stimulus, but also on the amplification, displayed time base, averaging procedures, and filtering of the recorded activity. ${ }^{213}$ In eight children typical albino VERs were recorded, as described by Creel et $a l^{28}$ and Apkarian et $a l^{13}$ with no difficulty in classifying them as asymmetric.

In four children, however, we recorded less but distinct asymmetries. In a study of two brothers, Summers et al ${ }^{25}$ showed asymmetric VERs in both, but only one had subnormal vision. Both had foveal changes and iris transillumination. The VER asymmetry of the brother with normal visual acuity was less distinct when compared with his brother's VER. This might be explained by a difference in the number of optic nerve fibres decussating in the optic chiasm. It is thus likely that, despite detailed procedure and apparatus, small asymmetries will not be detected (perhaps unless recordings are performed from the optic tracts). In our study we had two pairs of sisters (Table 1 ; patients 1 and 7 and patients 3 and 4) and they all have a pronounced iris transillumination and pathological maculas. However, one sister has an asymmetric VER while the other has a symmetric VER. We also have a pair of cousins (Table 1; patients 9 and 11). They both have a quite pronounced iris transillumination but normal maculae and their VERs are differently classified. Another interesting finding is that the mother of the child represented in Figure 3 also shows asymmetries, but restricted to a short time window indicating albinism (Fig 4).

Because of the anatomy of the visual pathways classic albinos are considered to lack stereopsis, ${ }^{5}$ but stereopsis is present in many VER asymmetric albinos. ${ }^{26}$ Moreover, in the report by Summers et $a l_{,}{ }^{25}$ the brother with iris transillumination, absence of foveal development, and asymmetric VER had good visual acuity (20/20) and stereopsis (100", TNO). Four of our eight patients with clear asymmetric VERs had good stereopsis (30-120"), one had fairly good stereopsis (240"), while three had stereopsis of $800^{\prime \prime}$ or less. One of these three patients had an esotropia.

Bergsma and Kaiser-Kupfer described a 'new' form of albinism ${ }^{7}$ with diffuse, fine, punctate transillumination of the iris, loss of foveal reflex, and mildly reduced visual acuity. They also found a diffuse, punctate depigmentation in the retina. VER recordings were not presented in these patients. Jay et al consider this form of albinism to be identical to the heterozygous state of oculocutaneous albinism but these patients usually have normal visual acuity. ${ }^{4}$ Our patients are in some ways similar to the patients described by Bergsma and Kaiser-Kupfer but the transillumination seems to be more coarse and they do not all have a loss of foveal reflex. The visual acuity is in the range $0.5-1.0$ but as a group the visual acuity is clearly subnormal compared with the control group. In fact, the first inclusion criterion was that these children were examined because of subnormal visual acuity during the ophthalmic section of the general health screening in Sweden at 4 years of age. In our study group there is a correlation between visual acuity and macular pathology. The patients with pathological maculas had best corrected visual acuity 
in the better eye of $0.5-0.7$. For the patients with normal maculas the corresponding acuity was $0.7-1.0$. If the foveal pigmentation is essential for the normal acuity development ${ }^{10} 12$ the lack of macular pigment in these patients could explain this difference.

There are several other plausible explanations for a subnormal visual acuity apart from albinism; for example, refractive errors, other malformations of the eye and optic nerve, retinal dystrophies, congenital nystagmus, delayed visual maturation (DVM), and mental retardation. During the examinations we have found one possible minor malformation, a pigmentation of unknown aetiology close to the papilla. However, the visual acuity in this eye has followed the fellow eye and is now 1.0. We have also found clearly indistinct maculas in seven patients but this could, or is likely to, be associated with albinism. None of the patients had a typical congenital nystagmus-that is, purely horizontal. ${ }^{6}$ Two patients had a very discrete rotatory nystagmus which should not alone affect the visual acuity. DVM is a retrospective diagnosis but the delay and improvement is usually seen during the first or at least during the second year of life. All our patients are older than this but it may exist as other forms of DVM/SVAS which improves later in life. None of the patients in the study group is mentally retarded or otherwise delayed in development.

Thus, SVAS seems to be a heterogeneous condition of high variability, including forms with chiasmal fibre decussation of almost normal rate, $50 \%$, to a maximal decussation of $100 \%$ of the optic axons. Vision may be normal, despite VER asymmetries. In our study group all had iris translucency as a sign of hypopigmentation and low acuity seems also weakly correlated with foveal abnormality. We believe that our patient group has some sort of albinism and that it is worthwhile looking for iris transillumination when visual acuity does not improve as expected. The albino diagnosis, with VER recordings if possible, can reassure the parents and patients that the condition is stationary or might improve and that they do not need to worry about deteriorating visual function.

In summary, there is no simple correlation of the degree of iris transillumination, stereopsis, visual acuity, and VER asymmetries. There seems to be a correlation between marked iris transillumination in all quadrants, absence of a foveal reflex, and low visual acuity (seven patients). In this group the visual acuity is $0.5-$ 0.7 in the better eye. Two patients with marked transillumination in all quadrants but normal maculae have a visual acuity of $0.9-1.0$ in the better eye. Patients with less transillumination (grade IV in two quadrants or less) all have normal maculae and visual acuity in the better eye $0.7-1.0$. Note also that VER asymmetry is not correlated with absence of stereopsis (Table 1).

This study was supported by The Blinda Vanners Foundation in Göteborg, Margit Thyselius Foundation for blind youth, Bohuslandstingets FoU-group, Kronprinsessan Margarethas arbetsnämnd, and Swedish Medical Research Council 02226.

1 Abadi R, Pascal E. The recognition and management of albinism. Ophthalmic Physiol Opt 1989; 9:3-15.

2 Creel JD, Summers CG, King RA. Visual anomalies associated with albinism. Ophthalmic Paediat Genet 1990;11:193200.

3 Guillery RW. Neural abnormalities of albinos. Trends in Neuro Sciences 1986;9:8.

4 Jay B, Carruthers J, Treplin M, Winder A. Human albinism. Birth defects. XII; no 3:415-26.

5 Kinnear PE, Jay B, Witkop JR CJ. Albinism. Surv Ophthalmol 1985;30.

6 Taylor D. Pediatric ophthalmology. Oxford: Blackwell Scientific Publications 1986:597.

7 Bergsma D, Kaiser-Kupfer M. A new form of albinism. Am Bergsma D, Kaiser-Kupfer M. A

8 Creel D, Witkop CJ Jr, King RA Asymmetric visually evoked potentials in human albinos: evidence for visual system anomalies. Invest Ophthalmol 1974;13:430-40.

9 van Dorp DB, van Haeringen NJ, Delleman JW, Apkarian P, Westerhof W. Albinism: phenotype or genotype? Doc Ophthalmol 1983;56:183-94.

10 Strongnin C, Guillery RW. The distribution of melanin in the developing optic cup and stalk and its relation to cellular degeneration. $₹$ Neurosci 1981;1193-204.

11 Silver J, Sapiro J. Axonal guidance during development of the optic nerve: the role of pigmented epithelia and other the optic nerve: the role of pigmented epithelia and

12 Abadi RV, Pascal E. Visual resolution limits in human albinism. Vision Res 1991;31:1445-7.

13 Apkarian P, Reits D, Spekreijse H, van Dorp D. A decisive electro-physiological test for human albinism. Electroencephalogr Clin Neurophysiol 1983:55:513-31.

14 Fitzgerald A, Billson F. Dissociated vertical deviation: evidence of abnormal visual pathway projection. $B r \mathcal{F} O p h$ thalmol 1984;68:801-6.

15 Kriss A, Timms C, Elston J. Visual evoked potentials in dissociated vertical deviation: a reappraisal. $\mathrm{Br} \mathcal{f}$ Ophthalmol 1989;73:265-70.

16 Apkarian P, Bour L, Barth PG. A unique achiasmatic anomaly detected in non-albinos with misrouted retinalangal projections. Eur $¥$ Neurosci (in press).

17 Khler L, Stigmar G. Vision screening of four-year-old children. Acta Paediatr Scand 1973;62:17-27.

18 Abrams JD. Transillumination of the iris during routine slitlamp examination. Br $f$ Ophthalmol 1964;48:48-4.

19 Laatikainen L, Erkkila H. Refractive errors and other ocular findings in school children. Acta Ophthalmol 1980;58:129 36.

20 de Vries J. Anisometropia in children: analysis of a hospital population. Br $\mathcal{F}$ Ophthalmol 1985;69:504-7

21 Fledelius $\mathrm{H}$. Prevalences of astigmatism and anisometropia in adult Danes. Acta Ophthalmol 1984;62:391-400

22 Sjöström A, Abrahamsson M. Patterned light flash evoked short latency activity in the visual system of visually normal and amblyopic subjects. Acta Physiol Scand (in press).

23 Sjöström A, Conradi NG, Andersson SA. Functional development of the visual system in normal and proteindeprived rats. V. Specific cortical response and repetitive deprived rats. V. Specific cortical response and repetitive stim.

24 Sjöström A, Abrahamsson M, Helgason G, Roos A, Norrsell EK. Flashed pattern induced activity in the visual system. I The evoked response recorded from the primary visua cortex of cat. Acta Physiol Scand 1991;143:1-9.

25 Summers CG, Creel D, Townsend D, King RA. Variable expression of vision in sibs with albinism. Am $\mathcal{f}$ Med Gene 1991; 40:327-31.

26 Apkarian P, Reits D. Global stereopsis in human albinos. Vision Res 1989;29:1359-70 